\title{
Toy model for the acceleration of blazar jets
}

\author{
I. Liodakis \\ KIPAC, Stanford University, 452 Lomita Mall, Stanford, CA 94305, USA \\ e-mail: ilioda@stanford.edu \\ Received 3 February 2018 / Accepted 20 April 2018

\begin{abstract}
Context. Understanding the acceleration mechanism of astrophysical jets has been a cumbersome endeavor from both the theoretical and observational perspective. Although several breakthroughs have been achieved in recent years, on all sides, we are still missing a comprehensive model for the acceleration of astrophysical jets.

Aims. In this work we attempt to construct a simple toy model that can account for several observational and theoretical results and allow us to probe different aspects of blazar jets usually inaccessible to observations.

Methods. We used the toy model and Lorentz factor estimates from the literature to constrain the black hole spin and external pressure gradient distributions of blazars.

Results. Our results show that (1) the model can reproduce the velocity, spin and external pressure gradient of the jet in M 87 inferred independently by observations; (2) blazars host highly spinning black holes with $99 \%$ of BL Lac objects and $80 \%$ of flat spectrum radio quasars having spins $a>0.6$; (3) the dichotomy between BL Lac objects and flat spectrum radio quasars could be attributed to their respective accretion rates. Using the results of the proposed model, we estimated the spin and external pressure gradient for 75
\end{abstract} \\ blazars.
}

Key words. galaxies: active - galaxies: jets - BL Lacertae objects: general - relativistic processes

\section{Introduction}

Black holes (BHs) of all masses are capable of producing collimated relativistic plasma outflows called jets. These jets are most likely produced via the Blandford-Znajek mechanism (BZ mechanism, Blandford \& Znajek 1977) where the energy powering the jet is extracted from the spin of the BH. Although the first jet was discovered a century ago in M 87 (Curtis 1918), the structure and acceleration mechanism of astrophysical jets remains an important unanswered question and field of active research to this day. In recent years great progress has been made in both theoretical and observational perspectives. Progress in the former is due to the increasing ability of modern computers to handle complex and computationally demanding simulations, while in the latter due to new facilities pushing the boundaries of energy and angular resolution. However, although progress has been made in different individual fields, we are still missing a unifying scheme for the structure and acceleration of $\mathrm{BH}-$ powered jets. Frequently used assumptions for the structure of the jets involve cylindrical, conical, and parabolic geometries, while the velocity of the jet $\left(u_{j}\right.$, usually expressed in terms of the Lorentz factor $\left.\Gamma=\left(1-\left(u_{j} / c\right)^{2}\right)^{-1 / 2}\right)$ is often assumed to be constant throughout the jet. However, variability timescales from different regions of the jet would imply, in at least some sources, different beaming properties (e.g., Ghisellini et al. 2005) rendering the constant Lorentz factor scenario unlikely. Acceleration is therefore a necessary ingredient in the jet paradigm.

From the theoretical perspective thermal driving has been shown to be inadequate to explain the high $\Gamma$ seen in jets suggesting that they have to initially be magnetically dominated (Vlahakis \& Königl 2004; Vlahakis 2015). For magnetically dominated jets the external pressure from the surrounding medium has an important contribution to the acceleration process (Vlahakis 2015). This has also been demonstrated in analytical and numerical work by Komissarov et al. (2007, 2009) and Lyubarsky (2009, 2010). In Lyubarsky (2009, 2010) it is shown that the external pressure could be responsible for the collimation of Poynting dominated jets and that the collimation and acceleration can take place over large distances. The jets are efficiently accelerated in the "equilibrium" regime while the Poynting dominated jet is slowly converted to a matterdominated jet. Although the jet will only become fully matter dominated at much larger distances, the acceleration is likely to stop when the magnetization parameter is $\sigma \leq 1$ (Vlahakis \& Königl 2003; Vlahakis 2004; Lyubarsky 2009). In the equilibrium regime, the jet will expand with decreasing external pressure until the pressure becomes constant. Then the jet will transition to a cylindrical geometry. Similar results have been obtained in Komissarov et al. (2007, 2009) where the magnetically dominated jet is confined by external pressure with a power-law profile $\left(p \propto z^{-s}\right)$. The jet has a parabolic shape as long as the power-law exponent is $s<2$. For $s>2$ the jet geometry will change from parabolic to conical.

From the observational perspective several studies have concluded that the acceleration zone is located upstream from the radio core of the jet (thought to be a standing shock and the location at which the jet reaches its maximum Lorentz factor, e.g., Marscher 1995) approximately at $10^{5} R_{s}$ from the $\mathrm{BH}$, where $R_{s}$ is the Schwarzschild radius Marscher et al. (2008, 2010). Recent results on M 87 suggest that the jet has a parabolic profile and accelerated up to the Bondi radius (which marks the sphere of gravitational influence of the $\mathrm{BH}, \sim 5 \times 10^{5} R_{s}$ also the location of HST-1), 
and then transitions to a conical geometry (Asada \& Nakamura 2012; Nakamura \& Asada 2013; Asada et al. 2014). Similar results for the acceleration profile of M 87 have been obtained by wavelet analysis in Mertens et al. (2016). This transition is thought to be caused by different profiles of external pressure making HST-1 a potential recollimation shock (Stawarz et al. 2006; Levinson \& Globus 2017). Results for Cygnus A suggest similar characteristics in jet structure and acceleration profile. The jet of Cygnus A is consistent with being externally confined and magnetically driven with the acceleration region extending up to $10^{4} R_{s}$ (Boccardi et al. 2016).

In this work, motivated by these recent results, we present a simple yet comprehensive toy model for the acceleration of blazar jets. Our goal is to create a simple framework on which both theorists and observers can build on in order to address more complex aspects of astrophysical jets. In Sect. 2 we present the toy model. In Sect. 3 we apply our model to $\Gamma$ estimates of blazars and in Sect. 4 we discuss the findings and conclusions of this work. In the Appendix we discuss the possible application of the model to gamma-ray bursts (GRBs).

\section{Toy model}

Considering the points raised above the toy model we propose is as follows. The jets are initially magnetically dominated and confined by external pressure having an initial parabolic geometry while accelerated over a large distance from the BH. The jet is accelerated through conversion of magnetic to kinetic energy until the two reach equipartition. The gas within the Bondi radius is forced to move inwards due to the gravitational pull of the BH. As expected from spherical accretion, the density and temperature of the gas will increase towards the $\mathrm{BH}$ creating a power-law profile for the density, and hence the power-law profile of the external pressure necessary to confine the jet (Bondi accretion has been found to be consistent with the observed luminosity of M 87, Di Matteo et al. 2003). Outside the Bondi radius the gas is free to move in any direction, and thus the external pressure loses its profile and can no longer collimate the jet into a parabolic shape necessary for the acceleration. At the Bondi radius observations would suggest the existence of a recollimation shock (Asada \& Nakamura 2012; Asada et al. 2014), which in blazars would be the observed radio core of the jet (Daly \& Marscher 1988; Marscher 2008). The formation of the shock could be due to the difference in the pressure profile of the surrounding medium (Gómez et al. 1997; Barniol Duran et al. 2017). Such a shock is also expected to form if the external pressure gradient is $s<2$ (Komissarov \& Falle 1997). The shock is the location where the jet reaches its maximum Lorentz factor since: (1) after the shock the jet is no longer collimated in a parabolic geometry and cannot be efficiently accelerated; and (2) the standing shock will inevitably decelerate the flow. Beyond the Bondi radius we have adopted a conical geometry as suggested by observations (Asada \& Nakamura 2012; Asada et al. 2014, see Sect. 4). The overall characteristics of the toy model are summarized in Fig. 1. In the equilibrium regime (where the jet is efficiently accelerated) the Lorentz factor grows as

$$
\Gamma \approx\left(\frac{z}{\omega_{\mathrm{LC}}}\right)^{s / 4}
$$

where $z$ is the distance from the $\mathrm{BH}, s$ is the power-law index of the external pressure $\left(p \propto z^{-s}\right)$, and $\omega_{\mathrm{LC}}=c / \Omega=c / 0.5 \Omega_{\mathrm{h}}$ is the cylindrical radius of the light cylinder, where $c$ is the speed of

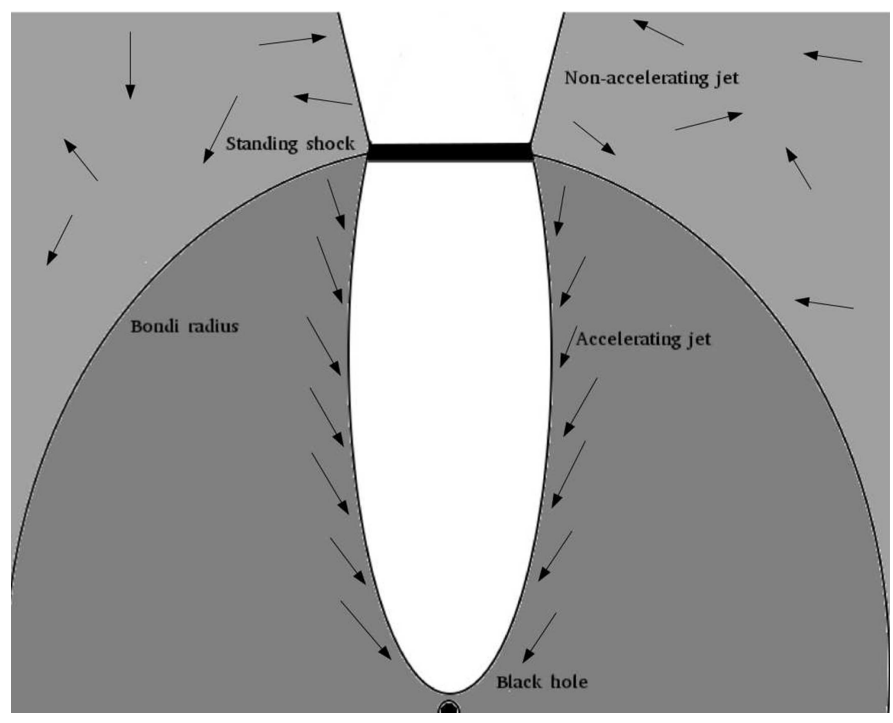

Fig. 1. Schematic of the toy model for the acceleration of astrophysical jets. The black arrows show the movement of the gas around the $\mathrm{BH}$ and jet.

light, and $\Omega_{\mathrm{h}}$ the angular velocity of the BH (Komissarov et al. 2009; Lyubarsky 2009; Boettcher et al. 2012 and references therein). According to the toy model the maximum $\Gamma$ is reached at the Bondi radius, i.e., $z=r_{\text {Bondi }}=2 G M / v_{\infty}^{2}$, where $G$ is the gravitational constant, $M$ the mass of the $\mathrm{BH}$, and $v_{\infty}$ the sound speed at the Bondi radius. Then,

$\Gamma_{\max }=\left(\frac{2 G M 0.5 \Omega_{\mathrm{h}}}{c v_{\infty}^{2}}\right)^{s / 4}$.

The angular velocity of the $\mathrm{BH}$ is defined as

$\Omega_{\mathrm{h}}=f_{\Omega_{\mathrm{h}}}(a) \frac{c^{3}}{2 G M}$

where $f_{\Omega_{\mathrm{h}}}(a)=a /\left(1+\sqrt{1-a^{2}}\right)$, and $a$ is the dimensionless spin of the $\mathrm{BH}$. Assuming a mean molecular weight $\mu=0.6$ and temperature $T=6.5 \times 10^{6} \mathrm{~K}$ (consistent with observations of M 87 , Narayan \& Fabian 2011; Russell et al. 2015) the sound speed at the Bondi radius becomes

$v_{\infty}=10^{-3} c$.

Combining Eqs. (2)-(4),

$\Gamma_{\max }=\left[5 \times 10^{5} f_{\Omega_{\mathrm{h}}}(a)\right]^{s / 4}$.

Equation (5) is independent of the BH mass which is a necessary condition since similar mass BHs in different systems (i.e microquasars and GRBs) produce jets with up to two orders of magnitude different $\Gamma$. $\Gamma_{\max }$ depends only on the spin of the BH and the gradient of the external pressure, with $\Gamma$ having a stronger dependence on the latter. For example, assuming $s=a=0.9$, Eq. (5) yields $\Gamma_{\max } \approx 17.24$. For a $10 \%$ change in $a$ there is a $7.6 \%$ change in $\Gamma_{\max }$, while a $10 \%$ change in $s$ results in a $33 \%$ change in $\Gamma_{\max }$. Thus there is only a mild dependence of $\Gamma_{\max }$ on the spin. 


\section{Application to blazar jets}

Studies on the spin and external pressure gradient of beamed sources are extremely rare and in the majority of cases unfeasible. The only source will available estimates for all three parameters that enter Eq. (5) is M 87. Studies of M 87 have determined that the gradient of the external pressure has a power-law index of $s=0.6$ (Stawarz et al. 2006); the maximum velocity of the jet at HST-1 is $\Gamma_{\max }=7.21 \pm 1.12$ (Wang \& Zhou 2009); and the BH has a spin of $a \approx 0.98_{-0.02}^{+0.012}$ (Feng \& Wu 2017). Using any pair of the above parameters Eq. (5) would yield the third within the uncertainties. Thus the model can produce values consistent with all three observed properties of the jet of M 87 .

Although we lack estimates of the $a$ and $s$ for blazars, we were able to use their observed $\Gamma$ (under the assumption that it is equal to $\Gamma_{\max }$ ) to constrain the distributions of the spin and the gradient of the external pressure. There are 75 blazar jets with available $\Gamma$ estimates (Hovatta et al. 2009; Liodakis et al. 2017; hereafter H09 and L17 respectively) 18 of which are BL Lac objects (BL Lacs) and 57 are flat spectrum radio quasars (FSRQ). These estimates are derived using variability Doppler factors (H09, L17) and apparent velocity estimates (Lister et al. 2009, 2013).

We assumed a distribution for $a$ and $s$ and use the observed $\Gamma_{\max }$ of blazars to constrain the optimal parameters for these distributions using a chi-square $\left(\chi^{2}\right)$ minimization procedure. For $a$, since it is bounded between $[0,1]$ a beta distribution is the natural choice ${ }^{1}$. For $s$ we tested a normal, a log-normal and a uniform distribution. The distributions (and their parameters) that yielded the lowest reduced $\chi^{2}$ value are shown in Table 1 . The results of the minimization were verified using the Kolmogorov-Smirnov $(\mathrm{K}-\mathrm{S})$ test $^{2}$. Figure 2 shows the cumulative distribution function for the observed and simulated $\Gamma_{\max }$ for both blazar populations.

The best-fit distributions for $a$ and $s$ are different for BL Lacs and FSRQs. For the spin, BL Lacs have generally larger spins with a mean of $\mu=0.937$ while FSRQs have a mean of $\mu=0.742$. It has been shown analytically that for spin values $a<0.6$ the $\mathrm{BZ}$ mechanism is no longer efficient (Maraschi et al. 2012). In order to account for the observed $\gamma$-ray emission of blazars Maraschi et al. (2012) constrained the spin of blazars to $a>0.5$, possibly as high as $a \sim 0.8$. Cosmological simulations of both BL Lacs and FSRQs have also determined that a sharp cut-off in the spin distribution of blazars is necessary in order to reproduce the number of observed sources in the Universe (Gardner \& Done 2014, 2018). Roughly $99.6 \%$ of BL Lacs and $80.5 \%$ of FSRQs in our sample have $a>0.6$. In addition, BL Lacs peak at $a \sim 0.9$ and FSRQs at $a \sim 0.8$ showing that our model naturally reproduces the results from different energetic and cosmological perspectives.

For the gradient of the external pressure, the BL Lacs follow a normal, while the FSRQs a log-normal distribution. The FSRQ distribution is also centered at, and extends to, higher values. This would suggest that, on average, the environment in the vicinity of the BHs of FSRQs is denser and therefore more gasrich than the environment in the vicinity of the BHs in BL Lacs.

\footnotetext{
1 Although different $\mathrm{BHs}$ are generally expected to have different spins, given the mild dependence of $\Gamma_{\max }$ on $a$ we also tested a delta function for the spin. The best-fit $a$ for both populations is $a \approx 0.72$. Even with the fewer degrees of freedom, the beta distribution still yielded, albeit marginally, a better model according to the reduced $\chi^{2}$. The $\mathrm{K}-\mathrm{S}$ test also favors the beta distribution over the delta function.

2 The K-S test yields the probability of two samples being drawn from the same distribution. We do not reject the null hypothesis for any $p$-value $>5 \%$.
}

Table 1. Parameters of the best-fit distributions of $a, s$ for BL Lacs and FSRQs.

\begin{tabular}{lcccccc}
\hline \hline Class & Param. & $\mu$ & $\sigma$ & $\chi^{2}$ & $\begin{array}{c}\chi^{2} \\
(\%)\end{array}$ & $\begin{array}{c}\mathrm{K}-\mathrm{S} \\
(\%)\end{array}$ \\
\hline BL Lacs & & & & 0.04 & 97.8 & 95.6 \\
& $a$ & 0.937 & 0.074 & & & \\
FSRQs & $s$ & 0.65 & 0.25 & & & \\
& $a$ & 0.742 & 0.163 & & & \\
& $s$ & 0.885 & 0.175 & & & \\
\hline
\end{tabular}

Notes. columns: (1) class, (2) parameter, (3) mean, (4) standard deviation, (5) reduced $\chi^{2}$ value, (6) $p$-value of the reduced $\chi^{2}$, (7) $p$-value of the K-S test. In both cases $a$ follows a beta distribution and $s$ follows a normal distribution for the BL Lacs and a log-normal for FSRQs. For the beta distribution the $\mu$ and $\sigma$ are defined as $\mu=\alpha /(\alpha+\beta)$, $\sigma^{2}=\alpha \beta /\left[(\alpha+\beta)^{2}(\alpha+\beta+1)\right]$ where $\alpha, \beta$ are the shape parameters. For the log-normal distribution the $\mu$ and $\sigma$ are defined as $\mu=\exp \left(l o c+s c^{2} / 2\right)$, $\sigma^{2}=\left(\exp \left(s c^{2}\right)-1\right) \exp \left(2 l+s c^{2}\right)$ where $l, s c$ are the location and scale parameters respectively.

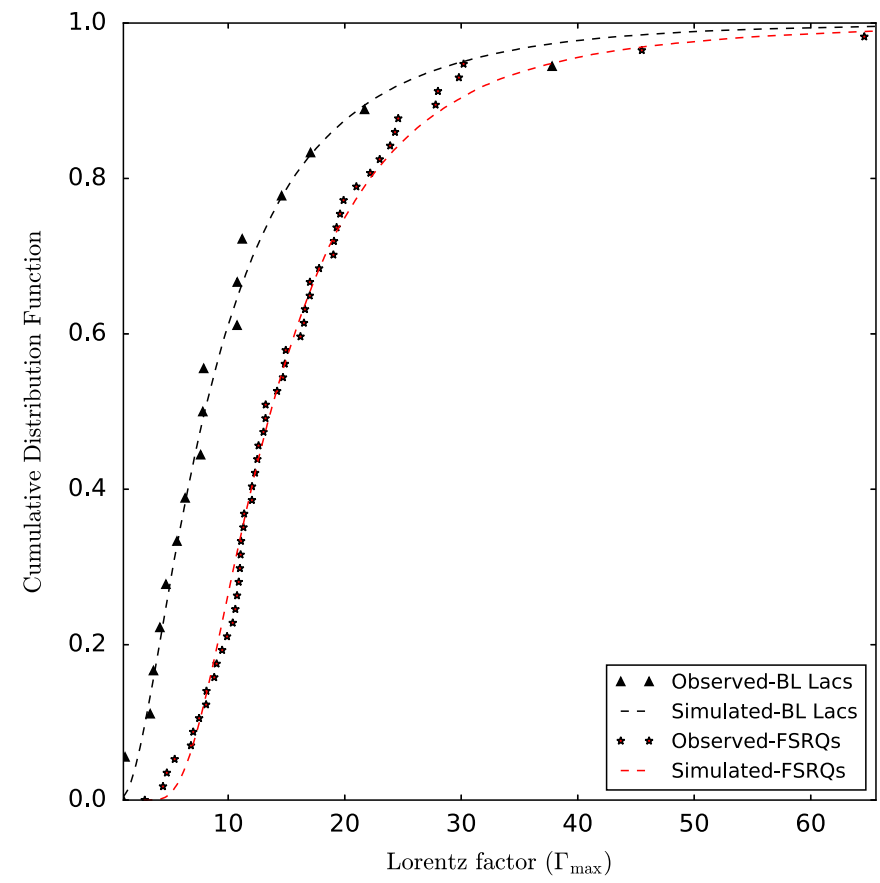

Fig. 2. Cumulative distribution function for the observed and simulated $\Gamma_{\max }$. Black triangles are for the observed BL Lacs and red stars for the observed FSRQs. The dashed black and red lines are the simulated sample for BL Lacs and FSRQs respectively.

Environmental conditions have been invoked in the past to explain the dichotomy between FR I and FR II type galaxies (the parent population of BL Lacs and FSRQs respectively). The results of the model would be consistent with evolutionary scenarios that attribute the differences in the two populations (BL Lacs \& FSRQs) to differences in their respective accretion rates (e.g., Böttcher \& Dermer 2002; Cavaliere \& D'Elia 2002; Ajello et al. 2014). If this is the case, then the fact that BL Lacs show on average higher spins would suggest that their BHs were spun up in the past either by accretion of gas that is now depleted (suggesting that BL Lacs are more evolved blazars than FSRQs) or by gas-poor mergers (e.g., Volonteri et al. 2005, 2007, 
Fanidakis et al. 2011) suggesting a different evolutionary track than FSRQs. The derived values for $s$ are swallower than predicted for Bondi accretion. They are, however, consistent with observations of M 87 (Stawarz et al. 2006) and are, for example, expected in the case were a ion torus supporting the jet is extending outwards from the supermassive BH (Rees et al. 1982).

In order to constrain the values for $a$ and $s$ for individual sources, we draw random values from the optimized distributions for $a, s$ for each population and minimize the square of the difference between observed $\Gamma_{\max }$ and the expectation from the toy model $\left(\left[\Gamma_{\mathrm{Obs}}-\Gamma_{\text {model }}\right]^{2}\right)$. Table B. 1 lists the optimal pairs of $a, s$ that reproduced the observed $\Gamma_{\max }$ after $10^{5}$ random draws. It should be noted that given the mild dependence of $\Gamma_{\max }$ on the spin, the values of $s$ are better constraint. Nearby sources with large viewing angles (e.g., J1221+2813, L17) could be used to test the predictions of the model for the gradient of the external pressure.

\section{Discussion and conclusions}

For the application of our model on blazars we used $\Gamma$ estimates from radio observations. These estimates were derived using a wide range of observing frequencies from 2.6 to $43 \mathrm{GHz}(\mathrm{H} 09, \mathrm{~L} 17)$. The radio frequency necessary to probe the region where the $\Gamma_{\max }$ is achieved dependents on the properties of source. Results from the MOJAVE survey would suggest that more than half of the blazar jets show accelerating features at $15 \mathrm{GHz}$ (Homan et al. 2015; Lister et al. 2016). Multiwavelength radio observations are then necessary to determine where and whether the $\Gamma_{\max }$ has been reached. For sources whose radio components show significant acceleration at the radio frequency where the Doppler factor (and hence $\Gamma$ ) was derived, the results of the model should be treated as lower limits.

Beyond the Bondi radius we have assumed that the jet has a conical geometry. This might not always be the case. Observations of M 87 do support that scenario (Asada \& Nakamura 2012; Asada et al. 2014). MHD simulations have also shown that beyond the recollimation shock at the Bondi radius the jet could become conical, however, depending on the pressure and density profile of the medium outside the Bondi radius different geometries are possible. The resulting geometry could have an impact on the velocity profile of the jet at large scales (e.g., Barniol Duran et al. 2017). Observations of additional AGN jet environments could give more insights on the fate of the jet beyond the Bondi radius.

Throughout this work, we have assumed that the earliest the jet would reach $\sigma \sim 1$ is at the Bondi radius. It is, however, possible for the jet to cease being Poynting dominated before reaching the radio core. In such a case the results of the model should be treated as upper limits. We have also assumed that the jet comprises of one bulk flow. It is possible that the Lorentz factor can also change transversely along the jet. Observations of high synchrotron peaked (HSP) sources in the TeV band have shown variability timescales which would require much larger Doppler factors than the ones derived from radio observations. In order to explain this discrepancy, Ghisellini et al. (2005) suggested a spine-sheath configuration: a fast inner spine responsible for the high-energy emission and a slower outer sheath. In this configuration, Ghisellini et al. (2005) found that the spectral energy distribution of four sources can be well described if the sheath has a $\Gamma=[3,3.5]$ and the spine a $\Gamma=[15,17]$. If such is the case for blazar jets then the radio observations (which probe the scales at which the $\Gamma_{\max }$ is reached) will be dominated by emission from the sheath
(Sikora et al. 2016). Although there are alternate hypothesis to the spine-sheath configuration that can fully explain the observed high energy emission without the need to invoke a faster bulk flow than the one derived from radio observations (e.g., magnetic reconnection, Giannios et al. 2009, 2010) the model can be easily extended to incorporate different flow configurations.

In this work we have presented a simple toy model for the structure and acceleration of jets from supermassive BHs and its application using observed $\Gamma$ estimates of blazars. Our findings can be summarized as follows:

- Application to M 87 showed that the model can produce consistent values with all three properties of the jet derived independently from observations.

- BL Lacs have on average higher spins than FSRQs, with both populations having the vast majority of sources with $a>0.6$ consistent with energetic considerations for the efficiency of the BZ mechanism as well as cosmological simulations.

- The results for the distribution of $s$ in BL Lacs and FSRQs would suggest that the BHs of the latter are, on average, in gas-richer and denser environments than the BHs of the former consistent with evolutionary models that attribute the differences of the two populations in their respective accretion rates.

Although there are many different aspects of the jets that have not been taken into account (MHD instabilities, energy conversion and dissipation mechanisms etc.), the fact that the model can produce consistent results with the observed properties of M 87 as well as the general properties of blazars would suggest that it is a good first approximation on which a more complex and realistic model could be built.

Acknowledgements. The author would like to thank the anonymous referee, Rodolfo Barniol Duran, Roger Blandford, Vasiliki Pavlidou, and Roger Romani for comments and discussions that helped improve this work.

\section{References}

Ajello, M., Romani, R. W., Gasparrini, D., et al. 2014, ApJ, 780, 73 Asada, K., \& Nakamura, M. 2012, ApJ, 745, L28

Asada, K., Nakamura, M., Doi, A., Nagai, H., \& Inoue, M. 2014, ApJ, 781, L2 Barniol Duran, R., Tchekhovskoy, A., \& Giannios, D. 2017, MNRAS, 469, 4957 Begelman, M. C. 2014, ArXiv e-prints [arXiv: 1410.8132]

Belczynski, K., Bulik, T., Fryer, C. L., et al. 2010, ApJ, 714, 1217

Blandford, R. D., \& Znajek, R. L. 1977, MNRAS, 179, 433

Boccardi, B., Krichbaum, T. P., Bach, U., et al. 2016, A\&A, 585, A 33

Boettcher, M., Harris, D. E., \& Krawczynski, H. 2012, Relativistic Jets from Active Galactic Nuclei (Berlin: Wiley)

Böttcher, M., \& Dermer, C. D. 2002, ApJ, 564, 86

Cavaliere, A., \& D'Elia, V. 2002, ApJ, 571, 226

Curtis, H. D. 1918, Publications of Lick Observatory, 13, 9

Daly, R. A., \& Marscher, A. P. 1988, ApJ, 334, 539

Di Matteo, T., Allen, S. W., Fabian, A. C., Wilson, A. S., \& Young, A. J. 2003, ApJ, 582, 133

Fanidakis, N., Baugh, C. M., Benson, A. J., et al. 2011, MNRAS, 410, 53

Feng, J., \& Wu, Q. 2017, MNRAS, 470, 612

Gardner, E., \& Done, C. 2014, MNRAS, 438, 779

Gardner, E., \& Done, C. 2018, MNRAS, 473, 2639

Ghisellini, G., Tavecchio, F., \& Chiaberge, M. 2005, A\&A, 432, 401

Giannios, D., Uzdensky, D. A., \& Begelman, M. C. 2009, MNRAS, 395, L29

Giannios, D., Uzdensky, D. A., \& Begelman, M. C. 2010, MNRAS, 402, 1649

Gómez, J. L., Martí, J. M., Marscher, A. P., Ibáñez, J. M., \& Alberdi, A. 1997, ApJ, 482, L33

Homan, D. C., Lister, M. L., Kovalev, Y. Y., et al. 2015, ApJ, 798, 134

Hovatta, T., Valtaoja, E., Tornikoski, M., \& Lähteenmäki, A. 2009, A\&A, 494, 527

Komissarov, S. S., \& Falle, S. A. E. G. 1997, MNRAS, 288, 833

Komissarov, S. S., Barkov, M. V., Vlahakis, N., \& Königl, A. 2007, MNRAS, 380,51 
I. Liodakis: Acceleration of astrophysical jets

Komissarov, S. S., Vlahakis, N., Königl, A., \& Barkov, M. V. 2009, MNRAS, 394, 1182

Komissarov, S. S., Vlahakis, N., \& Königl, A. 2010, MNRAS, 407, 17

Levinson, A., \& Globus, N. 2017, MNRAS, 465, 1608

Liodakis, I., Marchili, N., Angelakis, E., et al. 2017, MNRAS, 466, 4625

Lister, M. L., Cohen, M. H., Homan, D. C., et al. 2009, AJ, 138, 1874

Lister, M. L., Aller, M. F., Aller, H. D., et al. 2013, AJ, 146, 120

Lister, M. L., Aller, M. F., Aller, H. D., et al. 2016, AJ, 152, 12

Lyubarsky, Y. 2009, ApJ, 698, 1570

Lyubarsky, Y. E. 2010, MNRAS, 402, 353

Maraschi, L., Colpi, M., Ghisellini, G., Perego, A., \& Tavecchio, F. 2012, J. Phys. Conf. Ser., 355, 012016

Marscher, A. P. 1995, Proc. Nat. Acad. Sci., 92, 11439

Marscher, A. P. 2008, ASP Conf. Ser., 386, 437

Marscher, A. P., Jorstad, S. G., D'Arcangelo, F. D., et al. 2008, Nature, 452, 966

Marscher, A. P., Jorstad, S. G., Larionov, V. M., et al. 2010, ApJ, 710, L126

Mertens, F., Lobanov, A. P., Walker, R. C., \& Hardee, P. E. 2016, A\&A, 595, A54

Mészáros, P., \& Rees, M. J. 2001, ApJ, 556, L37

Nakamura, M., \& Asada, K. 2013, ApJ, 775, 118
Narayan, R., \& Fabian, A. C. 2011, MNRAS, 415, 3721

Piran, T. 2004, Rev. Mod. Phys., 76, 1143

Rees, M. J., Begelman, M. C., Blandford, R. D., \& Phinney, E. S. 1982, Nature, 295, 17

Russell, H. R., Fabian, A. C., McNamara, B. R., \& Broderick, A. E. 2015, MNRAS, 451, 588

Sapountzis, K., \& Vlahakis, N. 2013, MNRAS, 434, 1779

Schaerer, D., \& Maeder, A. 1992, A\&A, 263, 129

Sikora, M., Rutkowski, M., \& Begelman, M. C. 2016, MNRAS, 457, 1352

Stawarz, Ł., Aharonian, F., Kataoka, J., et al. 2006, MNRAS, 370, 981

Tchekhovskoy, A., McKinney, J. C., \& Narayan, R. 2008, MNRAS, 388, 551

Tchekhovskoy, A., Narayan, R., \& McKinney, J. C. 2010, New Ast., 15, 749

Vlahakis, N. 2004, Ap\&SS, 293, 67

Vlahakis, N. 2015, Astrophys. Space Sci. Lib., 414, 177

Vlahakis, N., \& Königl, A. 2003, ApJ, 596, 1104

Vlahakis, N., \& Königl, A. 2004, ApJ, 605, 656

Volonteri, M., Madau, P., Quataert, E., \& Rees, M. J. 2005, ApJ, 620, 69

Volonteri, M., Sikora, M., \& Lasota, J.-P. 2007, ApJ, 667, 704

Wang, C.-C., \& Zhou, H.-Y. 2009, MNRAS, 395, 301

Woosley, S. E. 1993, ApJ, 405, 273 


\section{Appendix A: Application to gamma-ray bursts}

Although the model has been constructed based on observations of jets from supermassive BHs, it would be interesting to explore its application to GRBs. Within the collapsar model (Woosley 1993), the stellar envelope could assume the role of the confining external medium collimating the flow. The jet would then be accelerated in a parabolic geometry until it breaks free from the star envelope to the ISM (Mészáros \& Rees 2001). According to Mészáros \& Rees (2001) $\Gamma$ would grow as $\Gamma \propto z^{1 / 2}$. If this is the case, our model can easily produce $\Gamma_{\max }$ of a few hundreds consistent with the $\Gamma$ seen in GRBs (e.g., Piran 2004; Begelman 2014). However, the toy model assumes that the end of acceleration takes place at the Bondi radius. In the case of GRBs the end of the jet acceleration would be at the radius $\left(R_{\star}\right)$ of the progenitor star. Then $z$ in Eq. (1) should be substituted with $R_{\star}, \Gamma=\left(0.5 R_{\star} f_{\Omega_{\mathrm{h}}}(a) c^{2} / 2 G M\right)^{s / 4}=\left(0.25 R_{\star} f_{\Omega_{\mathrm{h}}}(a) c^{2} k / G M_{\star}\right)^{s / 4}$, where $k$ is the mass ratio of the progenitor star to the resulting $\mathrm{BH}$ and depends on the metallicity of the progenitor (Belczynski et al. 2010). Using the mass-to-radius relation for Wolf-rayet stars, $R_{\star} / R_{\odot}=10^{-n}\left(M_{\star} / M_{\odot}\right)^{m}$, where $n=0.6629$ and $m=0.5840$ (Schaerer \& Maeder 1992) $\Gamma_{\max }$ becomes,

$\Gamma_{\max }=\left(\frac{0.25 c^{2} k 10^{-n / m}}{G} R_{\star}^{1-1 / m} f_{\Omega_{\mathrm{h}}}(a)\right)^{s / 4}$.

For similar metallicity progenitors $\Gamma_{\max }$ would depend on the spin of the resulting $\mathrm{BH}$, the pressure profile within the stellar envelope, and the radius of the progenitor star, $\Gamma_{\max } \propto\left(R_{\star}^{1-1 / m} f_{\Omega_{\mathrm{h}}}(a)\right)^{s / 4}$. RMHD simulations of GRBs have shown $\Gamma_{\max }$ to have the same dependences (Tchekhovskoy et al. 2008). However, it is also possible for the jet to experience rarefaction acceleration (Tchekhovskoy et al. 2010; Komissarov et al. 2010; Sapountzis \& Vlahakis 2013) when exiting the envelope of the progenitor star. If this is the case, the model could only be used to set the initial conditions of the rarefaction acceleration of the jet outside the progenitor star. The fact that the prediction of the model is in agreement with simulations shows some promise, although further investigation into whether this or a similar model is indeed applicable to GRBs is doubtless necessary.

\section{Appendix B: Spin and external pressure gradient estimates}

Table B.1. Spin and external pressure gradient estimates for blazars.

\begin{tabular}{|c|c|c|c|c|c|c|}
\hline Name & Alt-name & Class & $\Gamma$ & $a$ & $s$ & Ref. \\
\hline J0003-066 & NRAO 5 & $\mathrm{~B}$ & 3.3 & 0.9 & 0.38 & H09 \\
\hline J0016+731 & - & $\mathrm{F}$ & 6.8 & 0.59 & 0.64 & H09 \\
\hline $\mathrm{J} 0102+5824$ & $0059+5808$ & $\mathrm{~F}$ & 12.0 & 0.6 & 0.83 & L17 \\
\hline $\mathrm{J} 0106+013$ & OC 012 & $\mathrm{~F}$ & 27.8 & 0.82 & 1.07 & H09 \\
\hline J0136+4751 & $0133+476$ & $\mathrm{~F}$ & 9.5 & 0.49 & 0.76 & L17 \\
\hline $\mathrm{J} 0202+149$ & 4C 15.05 & $\mathrm{~F}$ & 9.9 & 0.61 & 0.76 & H09 \\
\hline $\mathrm{J} 0212+735$ & - & $\mathrm{F}$ & 7.5 & 0.57 & 0.67 & H09 \\
\hline $\mathrm{J} 0217+0144$ & PKS 0215+015 & $\mathrm{F}$ & 19.1 & 0.75 & 0.96 & L17 \\
\hline J0224+671 & - & $\mathrm{F}$ & 12.5 & 0.6 & 0.84 & H09 \\
\hline $\mathrm{J} 0237+2848$ & 4C 28.07 & $\mathrm{~F}$ & 14.9 & 0.75 & 0.88 & L17 \\
\hline $\mathrm{J} 0238+1636$ & $0235+164$ & B & 14.6 & 0.96 & 0.83 & L17 \\
\hline J0333+321 & NRAO 140 & $\mathrm{~F}$ & 14.7 & 0.9 & 0.85 & H09 \\
\hline J0336-019 & CTA 026 & $\mathrm{~F}$ & 23.0 & 0.78 & 1.01 & H09 \\
\hline $\mathrm{J} 0359+5057$ & $0355+50$ & $\mathrm{~F}$ & 13.2 & 0.75 & 0.84 & L17 \\
\hline J0423-0120 & PKS 0420-014 & $\mathrm{F}$ & 22.2 & 0.48 & 1.05 & L17 \\
\hline J0458-020 & PKS 0458-020 & $\mathrm{F}$ & 16.2 & 0.89 & 0.88 & H09 \\
\hline $\mathrm{J} 0530+1331$ & PKS 0528+134 & $\mathrm{F}$ & 10.8 & 0.86 & 0.76 & L17 \\
\hline J0552+398 & DA 193 & $\mathrm{~F}$ & 12.6 & 0.8 & 0.82 & H09 \\
\hline J0605-085 & PKS 0605-085 & $\mathrm{F}$ & 30.2 & 0.54 & 1.15 & H09 \\
\hline $\mathrm{J} 0642+449$ & $\mathrm{OH} 471$ & $\mathrm{~F}$ & 5.4 & 0.79 & 0.54 & H09 \\
\hline $\mathrm{J} 0721+7120$ & PKS 0716+714 & B & 10.8 & 0.93 & 0.75 & L17 \\
\hline J0736+017 & - & $\mathrm{F}$ & 17.0 & 0.8 & 0.91 & H09 \\
\hline $\mathrm{J} 0738+1742$ & $0735+178$ & B & 3.6 & 1.0 & 0.39 & L17 \\
\hline $\mathrm{J} 0754+100$ & OI 090.4 & B & 21.7 & 0.9 & 0.97 & H09 \\
\hline J0804+499 & - & $\mathrm{F}$ & 17.8 & 0.58 & 0.96 & H09 \\
\hline J0808-0751 & 0805-077 & $\mathrm{F}$ & 24.3 & 0.94 & 1.0 & L17 \\
\hline $\mathrm{J} 0818+4222$ & $0814+425$ & B & 4.1 & 0.93 & 0.45 & L17 \\
\hline $\mathrm{J} 0827+243$ & OJ 248 & $\mathrm{~F}$ & 23.9 & 0.62 & 1.05 & H09 \\
\hline J0836+710 & $4 \mathrm{C} 71.07$ & $\mathrm{~F}$ & 28.0 & 0.38 & 1.16 & H09 \\
\hline J0854+2006 & OJ 287 & B & 7.6 & 0.82 & 0.65 & L17 \\
\hline
\end{tabular}

Notes. Columns: (1) name as given in H09, L17, (2) alternative name, (3) class (B is for BL Lacs, F for FSRQs), (4) Lorentz factor, (5) spin, (6) external pressure gradient, (7) reference for the Lorentz factor estimate. 
I. Liodakis: Acceleration of astrophysical jets

Table B.1. continued.

\begin{tabular}{|c|c|c|c|c|c|c|}
\hline Name & Alt-name & Class & $\Gamma$ & $a$ & $s$ & Ref. \\
\hline J0920+4441 & S4 0917+449 & $\mathrm{F}$ & 2.8 & 0.67 & 0.37 & L17 \\
\hline $\mathrm{J} 0923+392$ & $4 C 39.25$ & $\mathrm{~F}$ & 4.4 & 0.53 & 0.5 & H09 \\
\hline $\mathrm{J} 0945+408$ & $4 C 40.24$ & $\mathrm{~F}$ & 29.8 & 0.76 & 1.1 & H09 \\
\hline J0958+6533 & $0954+658$ & $\mathrm{~B}$ & 7.9 & 0.98 & 0.64 & L17 \\
\hline $\mathrm{J} 1055+018$ & OL 093 & $\mathrm{~F}$ & 11.1 & 0.57 & 0.8 & H09 \\
\hline $\mathrm{J} 1104+3812$ & PKS $1101+384$ & $\mathrm{~B}$ & 1.1 & 1.0 & 0.04 & L17 \\
\hline $\mathrm{J} 1130-1449$ & $1127-145$ & $\mathrm{~F}$ & 13.0 & 0.26 & 0.93 & L17 \\
\hline $\mathrm{J} 1159+2914$ & PKS 1156+295 & $\mathrm{F}$ & 16.6 & 0.65 & 0.93 & L17 \\
\hline $\mathrm{J} 1221+2813$ & QSOB1219+285 & $\mathrm{B}$ & 4.6 & 0.99 & 0.47 & L17 \\
\hline $\mathrm{J} 1222+216$ & PKS $1222+216$ & $\mathrm{~F}$ & 45.5 & 0.57 & 1.28 & H09 \\
\hline $\mathrm{J} 1229+0203$ & $3 \mathrm{C} 273$ & $\mathrm{~F}$ & 12.0 & 0.69 & 0.82 & L17 \\
\hline $\mathrm{J} 1256-0547$ & 3C 279 & $\mathrm{~F}$ & 12.3 & 0.65 & 0.83 & L17 \\
\hline $\mathrm{J} 1310+3220$ & $1308+326$ & $\mathrm{~B}$ & 17.1 & 0.99 & 0.87 & L17 \\
\hline J1324+224 & - & $\mathrm{F}$ & 10.9 & 0.82 & 0.77 & H09 \\
\hline $\mathrm{J} 1332-0509$ & PKS 1329-049 & $\mathrm{F}$ & 11.1 & 0.39 & 0.83 & L17 \\
\hline $\mathrm{J} 1413+135$ & - & B & 6.3 & 0.98 & 0.57 & H09 \\
\hline $\mathrm{J} 1504+1029$ & OR 103 & $\mathrm{~F}$ & 11.4 & 0.7 & 0.8 & L17 \\
\hline $\mathrm{J} 1512-0905$ & PKS 1510-089 & $\mathrm{F}$ & 19.0 & 0.81 & 0.95 & L17 \\
\hline $\mathrm{J} 1538+149$ & 4C 14.60 & $\mathrm{~B}$ & 11.2 & 1.0 & 0.74 & H09 \\
\hline $\mathrm{J} 1606+106$ & $4 C 10.45$ & $\mathrm{~F}$ & 19.6 & 0.79 & 0.96 & H09 \\
\hline $\mathrm{J} 1611+343$ & DA 406 & $\mathrm{~F}$ & 14.2 & 0.71 & 0.87 & $\mathrm{H} 09$ \\
\hline $\mathrm{J} 1635+3808$ & $4 C 38.41$ & $\mathrm{~F}$ & 14.9 & 0.82 & 0.87 & L17 \\
\hline $\mathrm{J} 1637+574$ & OS 562 & $\mathrm{~F}$ & 11.0 & 0.63 & 0.79 & H09 \\
\hline $\mathrm{J} 1642+3948$ & $3 \mathrm{C} 345$ & $\mathrm{~F}$ & 11.3 & 0.53 & 0.82 & L17 \\
\hline $\mathrm{J} 1730-130$ & NRAO 530 & $\mathrm{~F}$ & 64.6 & 0.52 & 1.41 & H09 \\
\hline $\mathrm{J} 1751+0939$ & PKS 1749+096 & $\mathrm{B}$ & 7.8 & 0.98 & 0.64 & L17 \\
\hline $\mathrm{J} 1800+7828$ & S3 $1803+784$ & $\mathrm{~B}$ & 10.8 & 0.82 & 0.76 & L17 \\
\hline $\mathrm{J} 1807+698$ & 3C 371.0 & $\mathrm{~B}$ & 1.0 & 0.92 & 0.0 & H09 \\
\hline $\mathrm{J} 1823+568$ & $4 C 56.27$ & $\mathrm{~B}$ & 37.8 & 0.91 & 1.15 & H09 \\
\hline $\mathrm{J} 1828+487$ & 3C 380 & $\mathrm{~F}$ & 19.3 & 0.82 & 0.95 & H09 \\
\hline $\mathrm{J} 1848+3219$ & TXS $1846+322$ & $\mathrm{~F}$ & 7.0 & 0.39 & 0.68 & L17 \\
\hline $\mathrm{J} 1849+6705$ & S4 1849+670 & $\mathrm{F}$ & 17.0 & 0.92 & 0.89 & L17 \\
\hline J1928+738 & 4C 73.18 & $\mathrm{~F}$ & 19.9 & 0.79 & 0.96 & H09 \\
\hline $\mathrm{J} 2005+403$ & - & $\mathrm{F}$ & 21.0 & 0.88 & 0.97 & H09 \\
\hline J2025-0735 & PKS 2022-077 & $\mathrm{F}$ & 24.6 & 0.91 & 1.01 & L17 \\
\hline $\mathrm{J} 2121+053$ & - & $\mathrm{F}$ & 13.2 & 0.75 & 0.84 & H09 \\
\hline $\mathrm{J} 2134+004$ & OX 057 & $\mathrm{~F}$ & 9.0 & 0.67 & 0.72 & H09 \\
\hline $\mathrm{J} 2143+1743$ & PKS $2141+175$ & $\mathrm{~F}$ & 4.7 & 0.88 & 0.49 & L17 \\
\hline $\mathrm{J} 2201+315$ & 4C 31.63 & $\mathrm{~F}$ & 8.1 & 0.4 & 0.72 & H09 \\
\hline $\mathrm{J} 2202+4216$ & BL Lacertae & $\mathrm{B}$ & 5.6 & 0.97 & 0.53 & L17 \\
\hline $\mathrm{J} 2223-052$ & $3 \mathrm{C} 446$ & $\mathrm{~F}$ & 16.5 & 0.9 & 0.89 & H09 \\
\hline J2227-088 & - & $\mathrm{F}$ & 8.8 & 0.86 & 0.69 & H09 \\
\hline J2229-0832 & $2227-088$ & $\mathrm{~F}$ & 10.6 & 0.74 & 0.77 & L17 \\
\hline $\mathrm{J} 2232+1143$ & CTA 102 & $\mathrm{~F}$ & 8.1 & 0.46 & 0.72 & L17 \\
\hline $\mathrm{J} 2253+1608$ & $3 \mathrm{C} 454.3$ & $\mathrm{~F}$ & 10.4 & 0.77 & 0.76 & L17 \\
\hline
\end{tabular}

